\title{
Sharing is caring: the case for company level collaboration in pharmacoeconomic modelling
}

\author{
Anthony J Hatswell ${ }^{1,2}$ \& Fleur Chandler ${ }^{3,4}$ \\ 1 BresMed Health Solutions, Sheffield, UK \\ 2 Department of Statistical Science, University College London, UK \\ 3 Duchenne UK, London, UK \\ 4 GlaxoSmithKline, London, UK
}

Word count: $1187 / 1200$

In the recent NICE appraisals for rheumatoid arthritis the six companies involved each independently conducted systematic reviews, economic modelling and network meta-analyses [1]. Each will have incurred substantial agency fees and internal time to manage the projects. Given the models were also based on a very similar structure, each needed to solve very similar problems (independently) from the perspective of disease progression. This is a typical situation, with examples ranging from psoriasis, chronic obstructive pulmonary disease, and multiple myeloma with multiple companies creating almost identical models. In contrast, only a few examples exist of standardised models available to use by all (under varying frameworks [2,3]) - diabetes is the only example that readily springs to mind [4].

Whilst this may be the norm for our industry [5], we contend this does not represent a valuable use of scarce resources. Were companies to collaborate on modelling and evidence generation activities at the least at the disease level, there would be large benefits. These vary from improved modelling (reducing uncertainty), a broader evidence base, reduction of generating evidence which is duplicative or inconsistent, improved engagement with patients, lower costs and management time, additionally being ethically superior by making better use of patient input. We will discuss each of these areas in turn, as well as where collaboration is most likely to be advantageous.

Should a model be developed collaboratively by multiple companies, it would receive a much larger degree of scrutiny. Whilst this may take longer to achieve a final version, it also means mistakes are less likely to survive the process. With multiple companies also collaborating on model design, the resulting model is also more likely to generalise to the condition (rather than only apply to one treatment) - in that way the model is more likely to become accepted in the eyes of payers, and set the standard for that disease area, a benefit to the companies involved.

Companies are also likely to be running trials in different stages of the disease - multiple myeloma being a good example with some companies investigating first line treatments, others second, and others third and beyond. By pooling resources there would be a broader evidence base, with companies able to ensure the inputs used from outside their own trial are robustly captured. The benefits here are twofold; firstly in more reliable estimates of inputs, and secondly in reduced cost of evidence generation (as this is shared) - this can be seen as a saving, or used for further evidence generation.

By jointly developing a model, companies also have the potential to work with patient groups in a less transactional way. This can be used to get meaningful input - from the patient experience of the disease (and ensuring the model reflects this) to helping to populate health states (for example on working hours lost). Whilst this is possible for individual companies, it is less straightforward. Rare 
disease also only have a small pool of patients therefore a continuous stream of requests from different pharmaceutical companies may exhaust the goodwill of patients. Collecting data once (and comprehensively) in an effort to improve the modelling of the disease is preferable; such studies can then provide invaluable input to modellers and payers [6].

The lower cost of collaboration should also be addressed - whilst a more complex and adaptable model may cost more to construct, this is likely to be by a percentage, and not a factor. As the cost will be divided by at least 2 companies, in all cases this will result in a cost saving which increases as the number of collaborators increases. The true savings are much larger than they appear however for example, systematic reviews will only have to be completed once with no extra cost to be delivered to multiple companies whilst the internal time taken to manage contractors is a substantial element of the cost of modelling and would be reduced. With a contractor responsible for delivery to multiple companies, this element is shifted to the modelling agency (and explicitly costed in), rather than absorbed internally. This internal time can mean savings on staff time, or give time for other activities. The cost is then also borne by companies who have the greatest incentive to collect accurate data, rather than being contingent on a third party as proposed by Afzali et al. [7].

An additional factor that should be considered are the ethics of collaboration, something seldom relevant in health economics. When patients enter in to clinical trials of novel treatments, they put their future health at risk to advance medical science. Whilst complete data sharing between companies who may be competitors is not expected, the hard earned data for patients on placebo treatment, for example, could be by multiple companies in their development programs - the sacrifice and risk taken by patients is therefore honoured and used for the benefit of the patient community.

In practical terms the collaborative approach we propose at the company level is most likely to work in rare diseases, and in conditions where there have been no developments for many years. In rare conditions each company will be faced with the difficulty of understanding treatment pathways, and utilising scarce data to produce estimates - collaboration here is likely to help. Similarly in disease areas that have not had developments for some time (for example in melanoma prior to ipilumumab and vemurafenib [8]) similar problems apply as there is no best practice or established model, requiring a great deal of scoping and decision making. On the other hand collaboration will be more difficult where companies are on radically different timelines, or are likely to be competing for a finite pool of identical patients (for example the myriad of treatments for advanced or metastatic non-small cell lung cancer).

Whilst there are many benefits to collaboration, there are a number of issues to consider prior to companies entering in to agreements; briefly we believe these to be the extent of data sharing, how the model should be maintained in the future, how costs should be shared (particularly if companies have different timelines, or slightly different needs), and how the project should be managed. The legal implications of collaborating will also need to be carefully worded so as not to infringe rules on collusion (as price will be an input in models).

As a first step, Duchenne UK (a patient led charity) are currently proposing a collaboration between companies developing products in Duchenne muscular dystrophy (a rare muscle wasting disease affecting young boys). The disease pathway is complex, with current models not capturing important aspects of the condition and no data available for key inputs [9]. The goal of collaboration is to create a model which captures the entire disease pathway including joint research on the natural history of disease, quality of life data from all clinical trials, and populated with other information collected from patient groups (for example on carer burden and out of pocket costs). 
Ultimately whilst collaboration is not suitable for every area, we find the status quo wasteful. The current situation squanders time and money (which must be covered by increased prices, or lower returns), lowers quality (in turn increasing the chance of mistakes in resource allocation), increases uncertainty in modelling, and does not adequately respect the data generated from patients. When looking at upcoming projects, we urge companies to look beyond their own walls, and see if there is a way to do more with less - improving outcomes for all.

\section{Compliance with ethical standards}

AJH is an employee of BresMed Health Solutions, and FC an employee of GlaxoSmithKline. No funding was received for this manuscript, and the authors declare no conflicts of interest. 
1. National Institute for Health and Care Excellence. Final appraisal determination: Adalimumab, etanercept, infliximab, certolizumab pegol, golimumab, tocilizumab and abatacept for rheumatoid arthritis not previously treated with DMARDs or after conventional DMARDs only have failed [Internet]. National Institute for Health and Care Excellence; 2015 [cited 2017 Apr 19]. Available from: https://www.nice.org.uk/guidance/TA375/documents/rheumatoid-arthritis-adalimumabetanercept-infliximab-certolizumab-pegol-golimumab-abatacept-and-tocilizumab-review-id537final-appraisal-determination-document2

2. Sullivan W, Hirst M, Beard S, Gladwell D, Fagnani F, López Bastida J, et al. Economic evaluation in chronic pain: a systematic review and de novo flexible economic model. Eur. J. Health Econ. HEPAC Health Econ. Prev. Care. 2016;17:755-70.

3. Chilcott J, McCabe C, Tappenden P, O'Hagan A, Cooper NJ, Abrams K, et al. Modelling the cost effectiveness of interferon beta and glatiramer acetate in the management of multiple sclerosis. Commentary: evaluating disease modifying treatments in multiple sclerosis. BMJ. 2003;326:522; discussion 522.

4. Palmer AJ, Roze S, Valentine WJ, Minshall ME, Foos V, Lurati FM, et al. The CORE Diabetes Model: Projecting Long-term Clinical Outcomes, Costs and Costeffectiveness of Interventions in Diabetes Mellitus (Types 1 and 2) to Support Clinical and Reimbursement Decision-making. Curr. Med. Res. Opin. 2004;20:S5-26.

5. Arnold RJG, Ekins S. Time for Cooperation in Health Economics among the Modelling Community. PharmacoEconomics. 2010;28:609-13.

6. Payne KA, Varon SF, Kawata AK, Yeomans K, Wilcox TK, Manack A, et al. The International Burden of Migraine Study (IBMS): study design, methodology, and baseline cohort characteristics.

Cephalalgia Int. J. Headache. 2011;31:1116-30.

7. Afzali HHA, Karnon J. Addressing the Challenge for Well Informed and Consistent Reimbursement Decisions. PharmacoEconomics. 2011;29:823-5.

8. Lee D, Porter J, Hertel N, Hatswell AJ, Briggs A. Modelling Comparative Efficacy of Drugs with Different Survival Profiles: Ipilimumab, Vemurafenib and Dacarbazine in Advanced Melanoma. BioDrugs Clin. Immunother. Biopharm. Gene Ther. 2016;30:307-19.

9. National Institute for Health and Care Excellence. Final evaluation determination: Ataluren for treating Duchenne muscular dystrophy with a nonsense mutation in the dystrophin gene [Internet]. 2016 [cited 2017 Apr 19]. Available from: https://www.nice.org.uk/guidance/HST3/documents/finalevaluation-determination-document 